\title{
PREPROCESSING WITH SYMMETRICAL FACE AND GAMMA CORRECTION FOR FACE RECOGNITION UNDER VARYING ILLUMINATION WITH ROBUST REGRESSION CLASSIFICATION
}

\author{
${ }^{\mathrm{a}}$ Eva Y Puspaningrum, ${ }^{\mathrm{b}}$ Budi Nugroho, ${ }^{\mathrm{c}}$ Andri Istifariyanto \\ ${ }^{a, b}$ Faculty of Computer Science, University of Pembangunan Nasional Veteran JawaTimur \\ ${ }^{\mathrm{c}}$ Aviation Polytechnic of Surabaya \\ E-mail: evapuspaningrum.if@upnjatim.ac.id
}

\begin{abstract}
Facial recognition is one of the most popular issues in the field of pattern recognition. Face recognition with uncontrolled lighting conditions is more significant than the physical characteristics of individual faces. Uncontrolled lighting from the right and left can affect the face image. A lot of research on facial recognition, but little attention given to the face image is symmetrical object. Several studies to explore and exploit the symmetrical properties of the face for face recognition were performed. In this paper, we propose a pre-processing method to solve one of the common problems in facial images with varying illumination. We utilize the symmetric property of the face then performed gamma correction then classified using Robust Regression. The results of this experiment got an average accuracy of $94.31 \%$ and the proposed technique improves recognition accuracy especially in images with extreme lighting conditions using gamma correction parameters $\gamma=0.3$.
\end{abstract}

Keywords: Face Recognition, Symmetrical Face, Gamma Correction, Various Illumination 


\section{INTRODUCTION}

Face recognition is an active research topic and popular issues in the field of pattern recognition, computer vision, and machine learning. There are many methods to solve facial recognition problems especially to overcome the lighting conditions so that the normalization of illumination becomes an important but challenging research issue to improve facial recognition performance. Various lighting effects such as shadows, backwardness, and over exposure can cause images too dark or too bright, then bias the face recognition algorithm. It's well known that image are more variations of lighting changes is more significant than that due to different personal identity [1]. The degree of intensity variation on the face image may depend on the lighting conditions during image capture. For face images acquired under uncontrolled illumination conditions, non-uniform illumination problems arises [2]. The illumination correction approaches that correct for illumination problem and also retain the image feature characteristics in its original form are the illumination normalization approaches [3]. A number of preprocessing algorithms [4] to minimize the effect of illumination changes for face recognition have been developed, and many developments and advantages have occurred within the face model training stages. Illumination variation is the main obstacle for face recognition because face image appearances of the same person change under different illuminations. Sometimes, the changes of different illuminations among the same person are greater than those of different persons among the same illumination [5]. The Preprocessing method removes lighting effects without additional knowledge. Preprocessing based on image processing techniques transform images directly without assumptions or prior knowledge. Therefore, the preprocessing method can be used directly in the practical system for simplicity and efficiency [6]. Some of preprocessing method the widely used, due to their simplicity, such as histogram equalization (HE) [7], histogram specification (HS), logarithm transformation (LOG), logarithm transform (LT), self-quotient image (SQI) [8], new methods belonging to this category such as Gamma Intensity Correction
(GC) [9] have been proposed recently with impressive performance improvement for illumination problem.

Besides special algorithms are designed to overcome above difficulties, to obtain more face samples is another important way to improve the performance of face recognition [10]. For example, symmetrical face images proposed in [11] are very helpful to overcome the problem of varying poses and illuminations. Few methods take the facial geometry into account when generating more available face samples. When humans recognize faces, they often exploit the facial geometry information. A general property of most faces is that they have a symmetrically geometric structure. In other words, the right-half face is almost always the "mirror image" of the left-half face. This encourage to explore to exploit the symmetrical faces for face recognition algorithms [12].

In this paper, we propose a face image preprocessing method that use symmetrical face function and passes gamma correction to affect the brightness of images that have dark lighting. The data used is Yale Face Database $\mathrm{B}$, there is have face images of the same person under different conditions. One is taken in a controlled light while the other is captured in uncontrolled illumination conditions and from different angle. After preprocessing, In addition to this in this paper we classify of robust face recognition by formulating pattern recognition tasks as a problem of robust estimation. By using the basic concept that in general, the patterns from a single object class lie on a linear subspace to develop a linear model representing a probe as a linear combination of class specific galleries [13]. For the classification of methods used is Robust Linear Regression Classification (RLRC) algorithm is extensively evaluated for two important cases of robustness i.e. illumination variations and random pixel corruption [14].

\section{SYMMETRICAL FACE IMAGES}

We have followed a simple mathematical model for finding the symmetry. We have assumed face as a square depends on the dimension. In our experiment we have taken them as square. If we vertically bisect the 
square, it will be divided into two identical parts [15].

The proposed method to find the symmetry face is given below.

Step 1. Original matrix from image Yale Face Database B 50x50

Step 2. Center point analysis based on $\mathrm{x}$ axis Step 3. Image size identification to analyze the vertical line position to be used as mirror line

Step 4. Flip matrix image based on center point

Step 5. Sum Of original Matrix and new matrix value of the mirroring result

Step 6. Result matrix from merging process

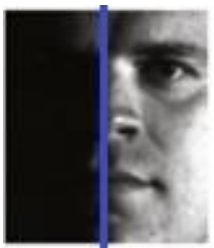

(a)

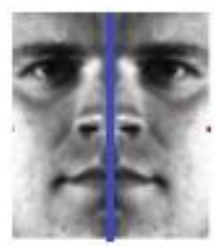

(b)
Figure 1 (a) Is one original image, (b) after the mirror image is done right in the middle of the frame

Figure 1 shows the symmetrical original face image and the facial image that the symmetric process has performed with the proposed method.

\section{GAMMA CORRECTION}

Gamma correction (GC) is a technique commonly used in the field of Computer Graphics. Gamma correction is a nonlinear operation used to control the overall brightness of a given image [2]. Images that are not properly corrected can look either bleached out, or too dark. Gamma correction can control the overall brightness of an image by changing the Gamma parameter [9]. At this point that the problem of the GC is due to the direction of light source on the illuminated object. And studies have shown that illumination effect due to changes in light direction can be addressed in the color domain when the source color is known and constant over a scene [2]. Gamma correction is defined as:

$$
I_{\text {output }}=I_{\text {input }}
$$

Gamma Correction is a gray level transformation that replaces each pixel with intensity $I$ in figure $I \gamma$. Where $\gamma$ is Gamma. Gamma value can be between the range $0 \leq \geq$ 1 .

\section{ROBUST REGRESSION CLASSIFICATION}

Face recognition by formulating pattern recognition using linear regression. Basic concept using linear regression is that the class patterns of a set of face images resides in a linear subspace, then a linear model of the test image is to be recognized as a linear combination of the training images [14]. This problem is solved by using the Robust Linear Regression method and the class determination is set based on Huber Estimation.

Consider a linear model

$$
a=X \beta+e
$$

Variable a is the dependent or response, the regressor or predictor variable $X$, the vector of parameters $\beta$, and error term e. The problem of robust estimation is to estimate the vector of parameters $\beta$ so as to minimize the residual

$$
b=a-\hat{a} ; \hat{a}=X \hat{\beta}
$$

$\hat{a}$ is the predicted response variable. In statistics the error term e is conventionally taken as a noise. Some approaches to robust estimation have been proposed such as Restimators and L-estimators. However, Mestimators have shown superiority due to their generality and high breakdown point. Primarily M-estimators are based on minimizing a function of residuals

$$
\hat{\beta}=\arg \min \left\{Y \hat{\beta}=\sum_{i=1}^{j} \rho(\gamma(\widehat{\beta}))\right\}
$$

Where $\rho(\gamma)$ is a symmetric function with a unique minimum at zero [14]:

$$
\rho(\gamma)=\left\{\begin{array}{c}
\frac{1}{2 \gamma} \gamma^{2} \\
|\gamma|-\frac{1}{2} \gamma
\end{array}\right.
$$

$\gamma$ is a tuning constant called the Huber threshold. Many algorithms have been developed for calculating the Huber M-estimate in Eq. (5). 
This method uses linear regression to solve facial recognition problems. The concept of this method is to have a class model of a set of facial images in a linear subspace. The linear model of the test image should be recognized as a linear combination of the training image. This problem is solved by using the Robust Regression Classification. To specify the class in a set of images then used Huber estimation. The face image will be represented as a smalldimensional vector in space $a x \quad b$. Small dimension vectors must be able to represent the face of each subject well. Two different facial images come from the same person [12]. The training phase in this method will produce a predictor for each class. For the dimension images $a \times b$ modified the grayscale process. The grayscale image will be converted to a down-sample process to produce a smaller size. The down-sample result is converted into a vector $w(i, m)$. Each vector image will be normalized so that the maximum value of pixels is 1 . Each class $i$ joins the vectors $w(i$, $m)$ to $X(i)$, as seen in Eq (6).

$$
X(i)=w(i, 1) w(i, 2) \ldots w(i, p(i))]
$$

where $p(i)$ is the amount of image data trained for class $i$. So it will produce a predictor for each class of $X(i)$.

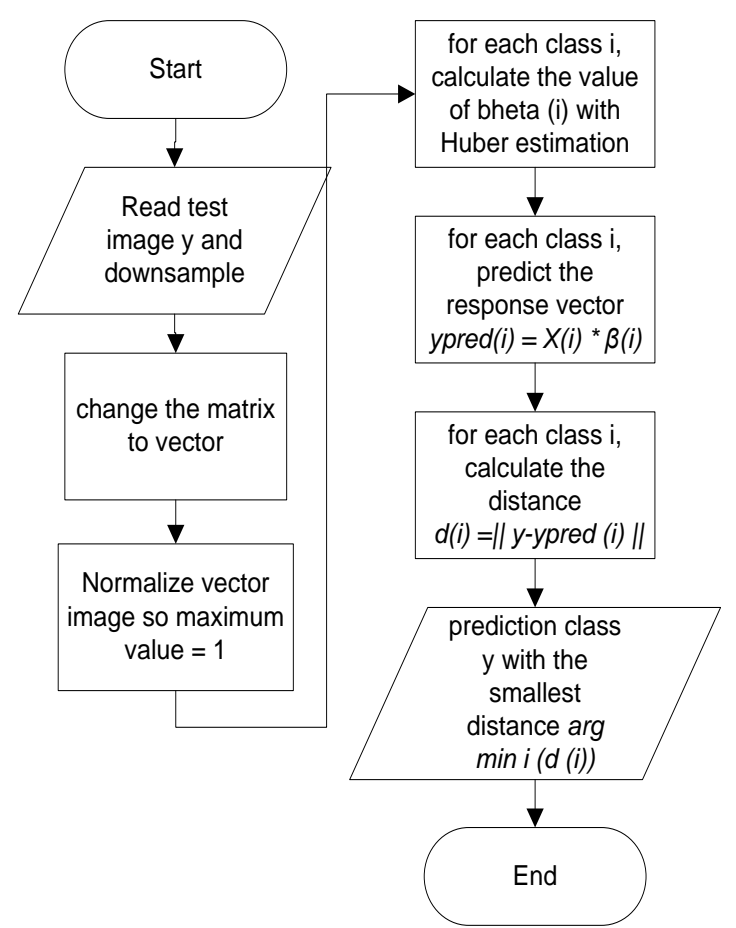

Figure 2. Flowchart Testing Process
The second stage is called testing process shown in Figure 2. Using the X predictor (i) of each class from the training stage results, a predictor matrix regression with the vector imaging test was performed using Huber estimation. For each class i, we will predict the response vector by using the predicted image ypred (i) $=X$ (i) * $\beta$ (i). For each class $\mathrm{i}$, estimate the value of $\beta$ (i) using the Huber parameter. This step aims to produce prediction image for image testing. After the prediction, then the predicted ypred (i) image will be calculated by the distance between the y input image and the predicted image ypred $(i)$ for each class i produced $d(i)=\| y$-ypred $(i) \|$. This predictive image which will predict the testing image class with the smallest distance. This step aims to generate prediction classes for testing images, based on the smallest distance between the vector images testing with a particular class prediction vector. So we will get the prediction of the image class y with the smallest distance $\arg \min i(d(i))$.

\section{RESULT AND DISCUSSION}

Yale Face Database B, there is have face images of the same person under different conditions. One is taken in a controlled light while the other is captured in uncontrolled illumination conditions and from different angle. The facial image on the dataset is the image of the face taken with straight position. Yale Face Database B 50x50 consisted of 10 individuals and 64 Illumination conditions per individual. There are several different scenarios to be done using this database. The Yale Face Database B 50x50 test scenario is based on the illuminated image angle shown in Table 1.

Table 1. Scenario Subset Yale Face Database B $50 \times 50$

\begin{tabular}{lccccc}
\hline $\begin{array}{l}\text { Subset } \\
\text { Image }\end{array}$ & 1 & 2 & 3 & 4 & 5 \\
\hline $\begin{array}{l}\text { Image } \\
\text { Number }\end{array}$ & $01-07$ & $08-19$ & $20-31$ & $32-45$ & $46-64$ \\
$\begin{array}{l}\text { Angle of } \\
\text { Degree }\end{array}$ & $0-2$ & $13-25$ & $26-50$ & $51-77$ & $>77$ \\
$\begin{array}{l}\text { Sum Of } \\
\text { Images }\end{array}$ & 7 & 12 & 12 & 14 & 19 \\
Scenario & Train & Test & Test & Test & Test \\
\hline
\end{tabular}


Of the scenario in Table 1, before the step of image classification with Robust Linear Regression will be done pre-processing with the proposed method by utilizing the symmetrical faces performed after mirroring. From the results of the new face image is done HE and GC. The results of the proposed preprocessing will be compared with the symmetry and HE.

The results of the experiment shown in Table 2. That shows that there is an increase in accuracy of facial recognition process using robust method that begins with pre-processing using the proposed method. Where the accuracy result from this research can be seen that the facial image with variation of illumination of the corners from Lighting Angle (degree) $51-77$ and $>77$ using this method look better.

Table 2. Recognition rates $(\%)$ on the Yale Face Database B 50x50

\begin{tabular}{cccc}
\hline $\begin{array}{c}\text { Subset } \\
\text { Image }\end{array}$ & $\begin{array}{c}\text { Original } \\
\text { Image } \\
\text { with HE }\end{array}$ & $\begin{array}{c}\text { symmetrical } \\
\text { face image } \\
\text { with HE }\end{array}$ & $\begin{array}{c}\text { symmetrical } \\
\text { face image } \\
\text { with HE + } \\
\text { GC }\end{array}$ \\
\hline Subset 2 & 100 & 100 & 100 \\
Subset 3 & 95 & 100 & 100 \\
Subset 4 & 72.10 & 93.56 & 93.57 \\
Subset 5 & 90 & 77.88 & 83.68 \\
\hline $\begin{array}{c}\text { Average } \\
\text { of }\end{array}$ & 89.27 & 92.86 & 94.31 \\
Accuracy & & & \\
\hline
\end{tabular}

\section{REFERENCES}

[1] A. K. Singh and G. C. Nandi, "Face Recognition Using Facial Symmetry," in Proceedings of the Second International Conference on Computational Science, Engineering and Information Technology, 2012, pp. 550-554.

[2] C. C. Chude-Olisah, G. Sulong, U. A. Chude-Okonkwo, and S. Z. Hashim, "Illumination Normalization for EdgeBased Face Recognition Using The Fusion of RGB Normalization and Gamma Correction," in Signal and Image Processing Applications (ICSIPA), 2013
An increase of accuracy can be seen in subset 4 and 5 where with the proposed method and using gamma correction parameter $\gamma=0.3$ improved accuracy. Increased accuracy shown in Table 2, increased accuracy using the proposed method at illumination angle 51-77 has an accuracy of $93.57 \%$ and $83.68 \%$ with angle $>77$. The average accuracy of all the subset tests using the proposed method has a better accuracy of $94.31 \%$ compared to the original image classification which has an accuracy of $89.27 \%$. Table 3 shows that the proposed method can improve face recognition results in straight and symmetrical facial data. Gamma correction helps increase the local dynamic range of images in the dark and compresses in bright areas. In experiments can be seen by adding gamma correction will affect the accuracy level. The proposed method consistently has high accuracy results for all experiments.

\section{CONCLUSION}

Based on the results of experiments that have been performed using Yale Face Database B 50x50 the proposed pre-processing method has an accuracy of increasing accuracy on subset 4 and 5 with a gamma correction parameter $\gamma=0.3$. From this experiment, increased accuracy using proposed method preprocessing at illumination angle 51-77 has an accuracy $93.57 \%$ and $83.68 \%$ with angle $>77$ degree.

IEEE International Conference on, 2013, pp. 412-416.

[3] B. Vinothkumar and P. Kumar, "Gamma Correction Technique Based Feature Extraction for Face Recognition System," vol. Vol. 3, No. 1, pp. 20-26, 2013.

[4] H. Wang, S. Z. Li, and Y. Wang, "Face Recognition Under Varying Lighting Conditions Using Self Quotient Image," in Automatic Face and Gesture Recognition, 2004. Proceedings. Sixth IEEE International Conference on, 2004, pp. 819-824. 
[5] I. Naseem, R. Togneri, and M. Bennamoun, "Robust Regression for Face Recognition," Pattern Recognit., vol. 45, no. 1, pp. 104-118, 2012.

[6] M.-C. Su and C.-H. Chou, "Application of Associative Memory in Human Face Detection," in Neural Networks, 1999. IJCNN'99. International Joint Conference on, 1999, vol. 5, pp. 3194-3197.

[7] R. Basri and D. W. Jacobs, "Lambertian Reflectance and Linear Subspaces," IEEE Int Conf Comput Vis, vol. 25, pp. 383-390, 2001.

[8] R. Ramamoorthi and P. Hanrahan, "On The Relationship Between Radiance and Irradiance: Determining The Illumination from Images of A Convex Lambertian Object," JOSA A, vol. 18, no. 10, pp. 2448-2459, 2001.

[9] S. Anila and N. Devarajan, "Preprocessing Technique for Face Recognition Applications Under Varying Illumination Conditions," Glob. J. Comput. Sci. Technol., 2012.

[10]S. Shan, W. Gao, B. Cao, and D. Zhao, "Illumination Normalization for Robust Face Recognition Against Varying Lighting Conditions," in Analysis and Modeling of Faces and Gestures, 2003. AMFG 2003. IEEE International Workshop on, 2003, pp. 157-164.
[11]S. M. Pizer et al., "Adaptive Histogram Equalization and Its Variations," Comput. Vis. Graph. Image Process., vol. 39, no. 3, pp. 355-368, 1987.

[12]W. Chen, M. J. Er, and S. Wu, "Illumination Compensation and Normalization for Robust Face Recognition Using Discrete Cosine Transform in Logarithm Domain," IEEE Trans. Syst. Man Cybern. Part B Cybern., vol. 36, no. 2, pp. 458-466, 2006.

[13] Y. Adini, Y. Moses, and S. Ullman, "Face recognition: The Problem of Compensating for Changes in Illumination Direction," IEEE Trans. Pattern Anal. Mach. Intell., vol. 19, no. 7, pp. 721-732, 1997.

[14]Y. Xu, Z. Zhang, G. Lu, and J. Yang, "Approximately Symmetrical Face Images for Image Preprocessing in Face Recognition and Sparse Representation Based Classification," Pattern Recognit. vol. 54, pp. 68-82, 2016.

[15]Z. Liu, J. Pu, Q. Wu, and X. Zhao, "Using the Original and Symmetrical Face Training Samples to Perform Collaborative Representation for Face Recognition,' Opt.-Int. J. Light Electron Opt., vol. 127, no. 4, pp. 1900-1904, 2016. 
